\title{
Enlarging knee masses
}

\author{
Hatem Mawi ${ }^{1}$ - Denis Gravel ${ }^{2} \cdot$ Joel Werier $^{3} \cdot$ Zaid Jibri $^{1}[$
}

Received: 16 April 2021 / Revised: 25 June 2021 / Accepted: 30 June 2021 / Published online: 11 July 2021 (c) ISS 2021

Question: A 67-year-old man presented with an 8-year history of progressive right knee swelling, increasing pain, and limited range of motion. MRI was performed (Fig. 1). Ultrasound-guided biopsy was obtained. Follow-up MRI was performed (Fig. 2), which was followed by surgical resection with the histopathologic examination (Fig. 3).

The diagnosis can be found at doi: 10.1007/s00256-021-03,860-2

Zaid Jibri

zaid.jibri@icloud.com

1 Department of Medical Imaging, Department of Radiology, The Ottawa Hospital, Ottawa, ON K1H 8L6, Canada

2 Division of Anatomical Pathology, The Ottawa Hospital, Ottawa, ON, Canada

3 Department of Surgery, The Ottawa Hospital, Ottawa, ON, Canada 
Fig. 1 MRI of the right knee including $\mathbf{a}$ and $\mathbf{b}$ sagittal T2

fat suppressed (FS), c sagittal proton density (PD), and $\mathbf{d}$ axial PDFS sequences
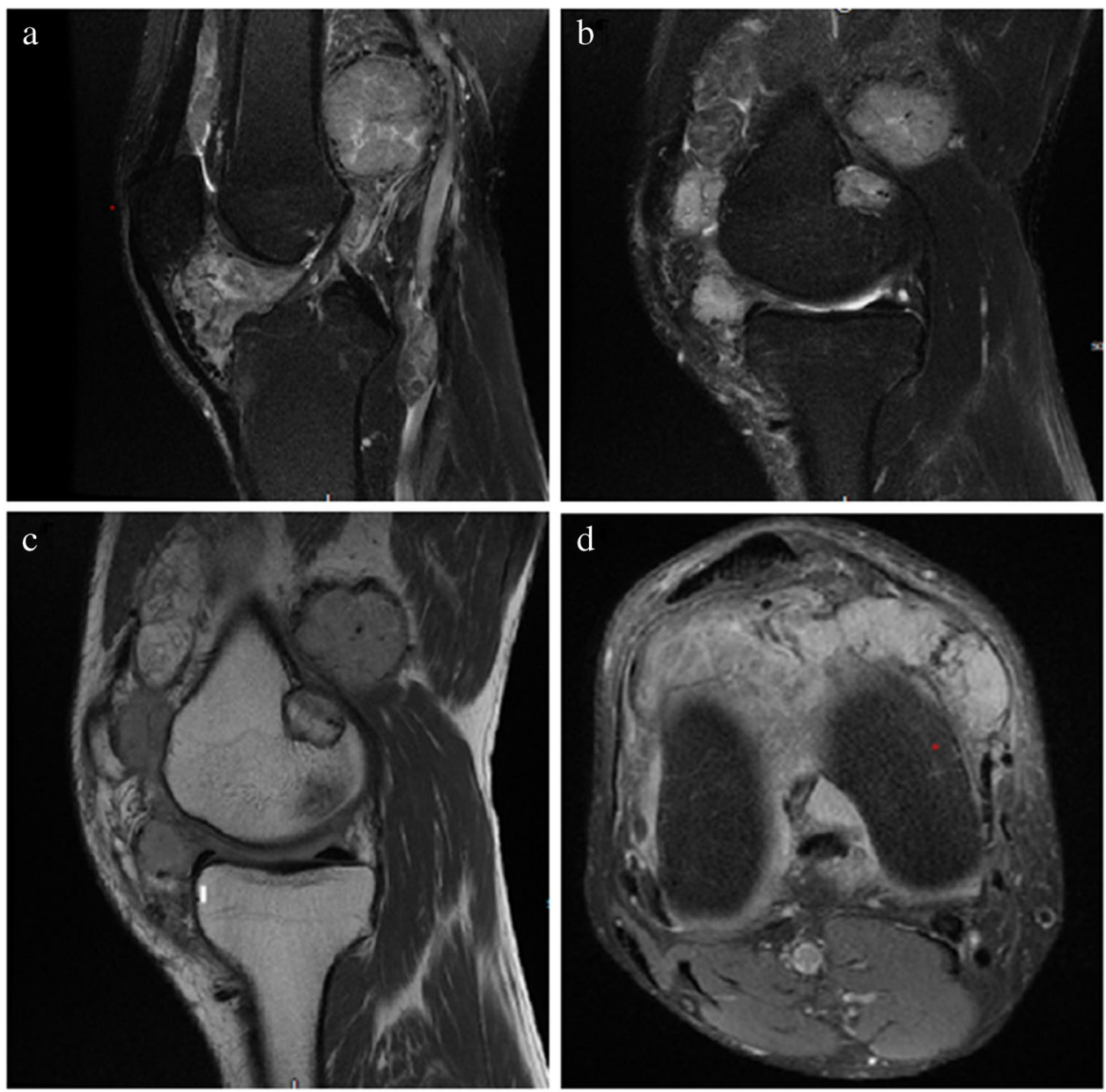
Fig. 2 Follow-up (20 months) MRI images of the right knee including a sagittal PD, b axial

$\mathrm{T} 1$, and $\mathbf{c}$ sagittal fat-suppressed $\mathrm{T} 1$ post-IV gadolinium

Fig. 3 Photomicrographs including hematoxylin and eosin $(\mathbf{a}-\mathbf{c})$, as well as immunohistochemical nuclear staining for STAT-6 (d)
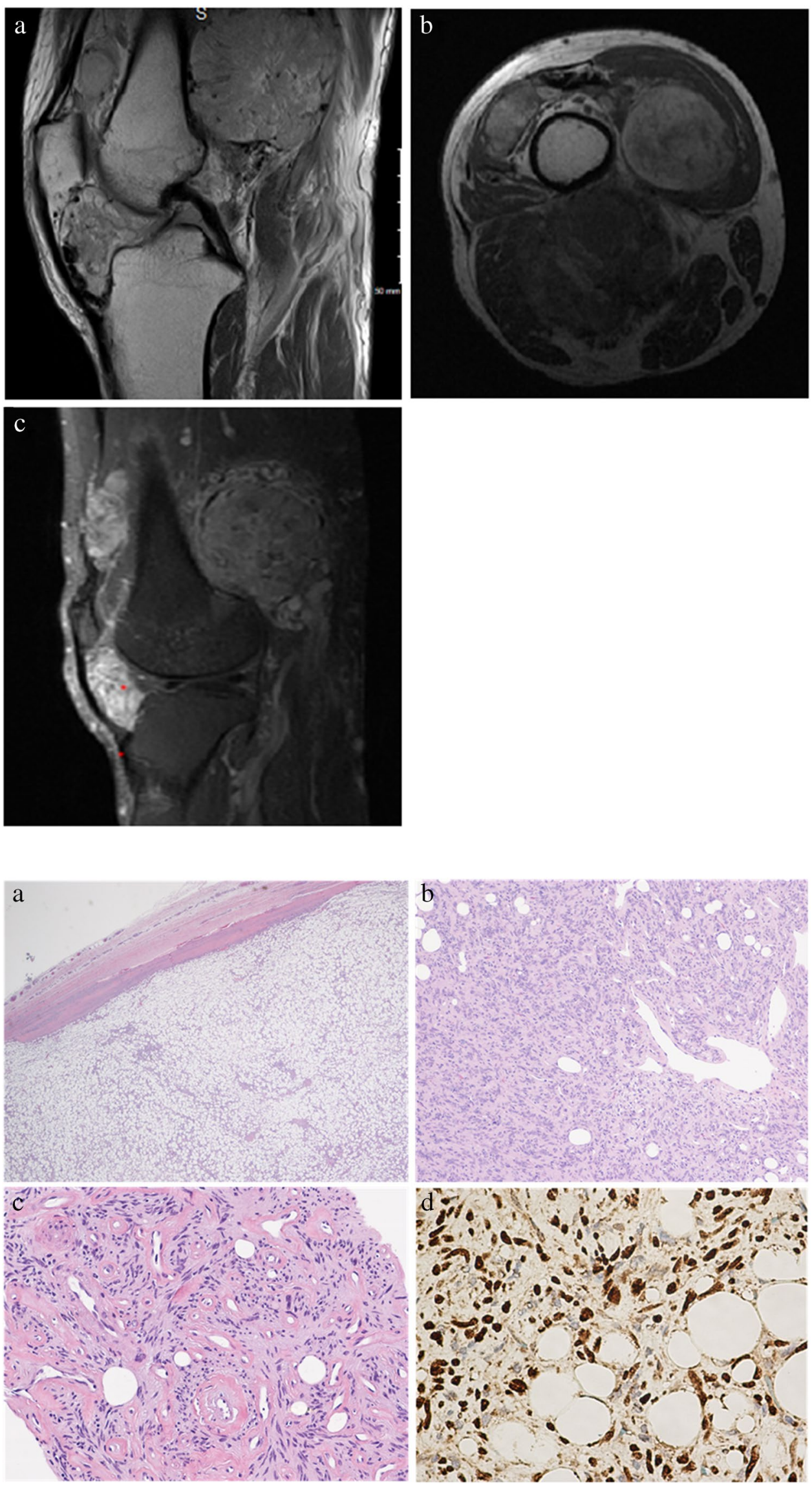


\section{Declarations}

Publisher's Note Springer Nature remains neutral with regard to jurisdictional claims in published maps and institutional affiliations.

Conflict of interest The authors declare no competing interests. 